\title{
Design and operation assessment of railway stations using passenger simulation
}

\author{
D. $\mathrm{Li}^{1} \&$ B. $\operatorname{Han}^{2}$

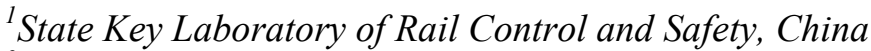 \\ ${ }^{2}$ Beijing Jiaotong University, China
}

\begin{abstract}
To assess the design of infrastructure and operation efficiency of railway stations, passenger simulation models are useful tools. This paper presents a microscopic passenger simulation model for railways. The simulation process is described as event planning, route choice and behaviour decision. Complex passenger behaviours are modelled, as well as simple motions. The model is calibrated using field data collected from Beijing railway station. Software called SRAIL is developed to validate the model. By using input passenger characteristics, station facilities, train timetables, traffic flow rules and simulation parameters, some useful indicators can be obtained. The indicators can reflect facility usage, delay, congestion, safety and coordination of the station. The total level of service is also evaluated. The first passenger dedicated railway station of the China - Beijing South Railway station is studied as an example. The result shows that the model can assess the station design and operation efficiently.
\end{abstract}

Keywords: railway station, design and operation assessment, microscopic passenger simulation, event planning, route choice, behaviour decision.

\section{Introduction}

The largest scale passenger dedicated railways are being constructed in China. Meanwhile, lots of new railway stations are being built. Most of these stations are passenger dedicated, modern designed, large scale, multi-floor structures and have a multi-modal traffic service. However, engineers are often faced with several problems: how to improve the efficiency of railway stations; how to avoid station travel time increasing time for the entire trip; how to keep large 
crowd passenger flows safe in the case of limited resources. Railway operators also hope to improve the level of service by using new technologies in new stations. They want to reduce the risk of operation accompanied by the lack of experience.

It is hard work to solve these problems through traditional methods, because there are many factors influencing station efficiency, such as passenger flows, passenger behaviour, layout of facilities and operation strategy. Moreover, the passenger crowd system is nonlinear; passenger flows on different facilities affect each other. Thanks to the development of simulation technology, by using passenger simulation, it is possible to forecast the potential problem of station facilities, operation schedule and emergency plan.

The paper is outlined as follows. In section 2, the literature is reviewed. In section 3, a passenger simulation model and its calibration is described. In section 4, the simulation tool SRAIL, based on the proposed model, is introduced. Section 5 is a case study of Beijing South Railway Station, which is the first passenger dedicated railway station in China. Finally, conclusions are provided.

\section{Literature review}

Traditionally, station assessment is done by mathematic method. The station is thought of as a cluster of facilities. By calculating the smallest capacity, the bottleneck is identified. However, the basic problem of capacity calculation is still not solved. Such method lasted for a long time, until the use of simulation in engineering. In particular, in the 1970s when Henderson [1] published the statistics of crowd fluids, many pedestrian simulation models were developed. The advantage of simulation is that the research object is modelled as an integrated system from passenger facility to operation strategy.

Although there are only a few researchers studying passenger simulation in railways, pedestrian simulation is widely studied, since it is a common technology. Many specialists from physics, civil engineering and social science have made great contributions in this field. Different methods were used to study pedestrian flows, such as computational physics, hydromechanics, cellular dynamics, artificial intelligence and society. However, much attention has been

Table 1: $\quad$ Researches review.

\begin{tabular}{|l|l|l|l|l|l|}
\hline Year & Researcher & Model & Year & Researcher & Tools \\
\hline 1985 & Gipps [3] & Benefit cost & 1990 & Maw [12] & PedRoute \\
\hline 1993 & Okazaki [4] & Gravity & 1998 & Gordge [13] & Station \\
\hline 1994 & Lovas [5] & Queue network & 1999 & Schelhorn [14] & STREETS \\
\hline 1994 & Rothman [6] & Lattice gas & 2000 & Still [15] & LEGION \\
\hline 1995 & Helbing [7] & Social force & 2003 & Steps [16] & STEPS \\
\hline 2000 & Hoogendoorn [8] & Gas kinetic & 2004 & Hoogendoorn [17] & Nomad \\
\hline 2000 & Blue [9] & CA & 2007 & Li [18] & SRAIL \\
\hline 2003 & Kirchner [10] & Floor Field & & & \\
\hline 2009 & Izquierdo [11] & PSO & & & \\
\hline
\end{tabular}


paid to theories until recently, when physicist Helbing's [2] book "Managing Complexity: Insights, Concepts, Applications" was published. Although the mechanism of pedestrian behaviour is not very clear, useful models and tools were developed. Typical researches are classified as theories and applications, which are listed in table 1.

Most of the above tools are used on egress, which has a simple flow. The most widely used tool is Legion, which is based on crowd dynamics. However, it is not especially designed for railway traffic. Many scenarios of railway station operation could not be effectively simulated. Nomad is the first tool specifically for railway. A systematic indicator set is proposed for assessing the railway station, as this is important to facility configuration. Despite a microscopic model, the simulation of complex systems, such as stations, need more detailed work. These include an activity model, route choice model, behaviour model, integrated model and so on.

\section{Modelling and calibration}

\subsection{Model hierarchy}

To assess the railway station design and operation, it should be very flexible on both infrastructure modelling and simulation dynamics. The model is divided into macroscopic, mesoscopic and microscopic levels (see fig. 1). At each level, models are set up for station facility, passenger and operation strategy. The advantage of this structure is any changes of station design or operation strategy are related to passenger behaviour, so the assessment of station facility design and operation efficiency can be more easily achieved.

(1) Station model. The station is defined as a graph $G(N, E)$ at macroscopic level. A node indicates functional blocks such as the railroad, bus, taxi, metro and park system. These nodes are the places where passengers "appear" or "disappear". A link is the connection between these systems. At mesoscopic level, facilities' relationship is described as a logic network. Facilities, such as escalators, staircases, concourses and platforms, are modelled as units with different properties so that the passenger can identify them. This level is also designed to deal with the connection between different floors. At microscopic level, each facility system is described as a grid with dynamic cell size. The movable passenger can occupy the cell, and have real time interaction with facilities through it.

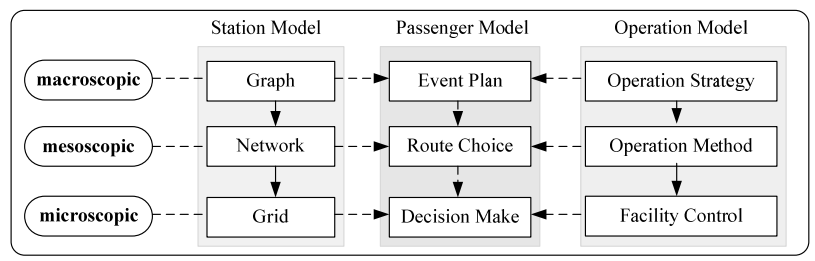

Figure 1: $\quad$ Model hierarchy. 
(2) Passenger model. The passenger is modelled as entities with three property groups. The first is basic properties, including physical properties (gender, age, body size, vision range, and walk-ability) and psychological properties (psychological distance, temper). The second is social properties, such as familiarity with environment, educational background, partners, attractiveness and trip experiences. The third is traffic properties, such as trip aim, origin, destination, desired speed, acceleration, position, ticket, and luggage size.

The passenger's process model in the railway station is classified into three steps, thus it can seamlessly interact with the station model. The three steps are event planning, route choice and decision-making. The event planning is as follows: when a passenger enters the station, he should clearly know what his aim is, then make an activity plan of what he would do in the station before leaving. The activity plan is highly related to the traffic aim and time need. To depart, passengers who have a long time before they leave might make a rich activity plan. In contrast, passengers who have little time or just arrive at the station would only do the necessary activities. The route choice is a process when the event is relatively determined. Passengers should try to find a reasonable target to achieve their aim. However, on most occasions, there is more than one target. Passengers should select a target that would maximize their utilities. The last step is decision making: the passenger should decide how he gets the target and which behaviour is reasonable. The decision is made according to the state of the passenger and the station. In this step, passenger behaviour modelling is also very important. Passenger behaviour is designed to have add-ons. It means users can develop their own behaviour models. Although different passengers would have different behaviours, they have some behaviour in common. In a railway station, behaviours are modelled, such as buying a ticket, waiting to board, queuing, checking in, looking at the information screen, alighting and boarding. These complex models are made up of simpler models, such as walking, obstacle avoidance, waiting, wandering, seeking and path following.

Actually, such a process is not always from top to bottom. Passengers might change their activities or decisions temporarily according to the situation they are confronted with. For example, passengers with a lot of spare time would adjust their activity, even their walking speed. Passengers who feel bored may wander here and there. Passengers who feel tension may try to get ahead of others in queues. In order to model the various activities, a dynamic activity network is established. The network is the description of all necessary activities. Passengers can either follow the network or separate from the network temporarily, as long as they do not deviate from the target. In fact, there are a lot of factors that may influence a passenger's planning and decision making process. Some are even not very clear. In this model, the user can define exactly which rule the passenger should obey at each level.

(3) Operation model. This manages the operation strategy of the station. By providing a user interface, many operation methods could be implemented from passenger flow line management to timetable adjustment. The result would affect the facility state of the station and behaviour of each passenger. It decides 
questions such as when the ticket gate opens, how long it takes to get a ticket and where should the train stop. For example, one can adjust the stop time of a train from the interface, or adjust the direction of the automatic fare gates. At service systems, it also controls the reasonable queue to make the simulation more realistic. Connections are also set up from the event and passengers model by the operation model. For example, a train arrival event, passenger generation event and facility state switch event may trigger at the same time.

\subsection{Four steps model calibration and validation}

It is important to perform a passenger simulation calibration before using the model, although it is more difficult to use than the general pedestrian simulation model. The model should not only reflect the basic passenger behaviours under different conditions, but also obey the fundamental diagrams of pedestrian flow. Moreover, the activity of the passenger and his time consumed in the station should be kept consistent with real operation. We present a four step calibration method to ensure the availability of the model.

(1) Fundamental diagram test. Passenger flow should obey traffic flow characteristics at macroscopic level, although individual behaviour might be completely different. Special experiments, such as passenger movement on loop facilities (a certain width corridor with unlimited length), are designed. After some warm up time, the passenger movement is simulated under a different crowd level. The density, flow and speed data is recorded. The relationship is compared with an empirical study of prior researchers, as shown in fig. 2.

It is found that the capacity flow is about $110 \mathrm{p} / \mathrm{min} / \mathrm{m}$ when space is $0.5 \mathrm{~m}^{2} / \mathrm{p}$. This is very close to the fundamental diagram of the HCM. The capacity value is also equal to the practical measurement in Beijing.

(2) Self organization test. One of the most famous characteristics of passenger flow is self organization phenomenon. Unlike other traffic modes, when the flow approaches the capacity or on other occasions, some special phenomena, such as lane formation, bubbles, bottleneck oscillations and moving stripes, can appear; this is not deliberately designed. Taking the bubbles and bottleneck oscillations as examples, the proposed model is tested. The bottleneck is set to a $0.5 \mathrm{~m}$ narrow

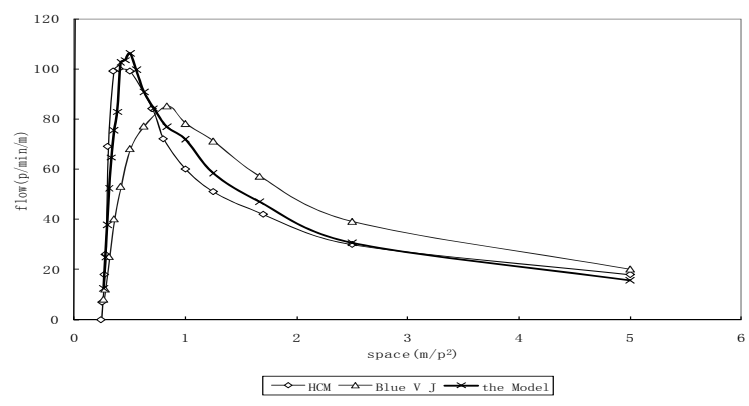

Figure 2: $\quad$ Space flow relationship of the model. 


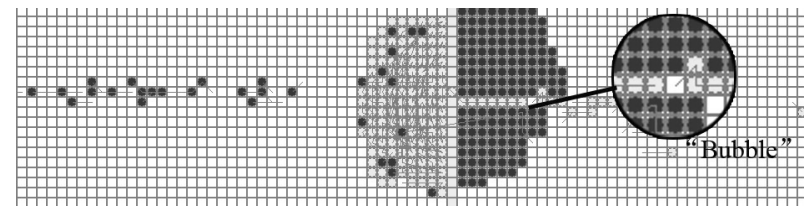

Figure 3: $\quad$ Bottleneck oscillations and bubbles.

door. When the bi-directional passenger flows pass the bottleneck during a large crowd scenario, instead of deadlock, passengers from one side pass the door first and after a while the opposite side, until the bottleneck has cleared. The result is shown in fig. 3.

This phenomenon is widely found in prior researchers' studies. In the test, the highest frequency found is about 45 seconds, which reduces multi-nominally with an increase of the door width. It is also found that even in very high densities, some spaces would not be effectively used. These spaces were called bubbles. This is consistent with the real world.

(3) Field data test. It is generally accepted that people's traffic behaviour is different under different areas, environment and cultures. It is also found that passengers' use of different facilities is very different. So it is necessary to validate the model using field data test.

Firstly, a data collector and analysis tool is developed for the validation. The video data is first collected from the CCTV in the station. Then each passenger's coordinates at different times are extracted from the video. The data relationship curves, for example for evacuation versus time and distance versus flow, are analyzed and compared with the simulation result in the same scenario.

Secondly, a special purpose survey is carried out, such as for time consuming investigation. Each surveyor would select a passenger randomly, and try to follow him. The surveyor would record the time of each activity and each target position. For example, at the entrance, ticket vendor, waiting room or gate. Other data, such as station structure, timetable and parameters, are also obtained from the station operation agency.

The simulation scenario is carefully imported in to the model and, after a $24 \mathrm{~h}$ simulation, the simulation data for time consumption is collected and analyzed. By comparing the result with the field data statistics, the model is validated or revised.

(4) Empirical formula test. Railway operators have summarized much useful "knowledge" about passenger flow, facility use and operation method. For example, the unidirectional flow is more effective than mixed flow, long distance corridors can ease passenger flow congestion and sometimes a set of obstacles might be useful to improve the safety of the flow. Besides, some empirical formulas were also given, such as the station egress time confirmation. Although these formulas are not absolutely correct, they reflect the effects of some factors relatively. The results with different input parameters should be consistent with some existing knowledge.

The model is tested with special experiments. For example, the escalator width is changed in different scenarios, while keeping other parameters the same. 
The empirical knowledge told us that the level of service is lower when the width is narrower, and passengers would rather gather at escalators than select the staircase. Some field data can also be collected to make the knowledge quantitative.

\section{The SRAIL system}

SRAIL is a passenger simulation system for railway stations, developed using the proposed model. The system is made up of a station editor module, data collector module, passenger flow generation module, passenger activity design module, passenger behaviour simulator, station service simulator, data replay module, 3D simulator, simulation data analyzer and auto-report system.

\subsection{Input}

The input of the system depends on how complicated the simulation scenario is. Basically, it includes the station facilities profile, passenger flow generation profile, activity profile and system parameters.

The station editor module provides a tool to edit station facilities, such as the entrance, exit, concourse, escalators, staircase, gate and platform. The user should also define the position and parameters of the station service.

The passenger flow generation system provides three types of model. Passengers could be generated by probability distribution, by train timetable or by OD-matrix. This is dependent upon how accurately the operators know the rule of passenger arrival flow.

The passenger activity profile gives the user the opportunity to change operation strategies. For example, in most of the railway stations in China, passengers should wait for the train before checking their ticket; this is called "wait first then check". However, new passenger dedicated railway stations reserved the "check first then wait" method. This can be edited conveniently by the activity module.

\subsection{Simulator}

The passenger behaviour simulator is the core simulator of the passengers' motion. According to the model, passengers' behaviours are determined. The station service simulator is also very important, because it controls the changeable facilities or services of the station. In the railway station, there might be a lot of service systems with queues or without queues. The station service simulator controls and updates the queue systems. In some stations, a ticket gate's open time is related to the train departure time, thus the simulator provides the connection between them. It can also maintain user defined service systems, such as a security check.

\subsection{Output}

The system provides a lot of useful indicators as output. Basically, it can be divided into three categories: quantity indicators, time indicators and integrated 


\begin{tabular}{|c|c|c|c|c|c|c|}
\hline \multicolumn{7}{|c|}{ Assessment Indicator System } \\
\hline Facility Use & \multicolumn{2}{|c|}{ Time \& Convenience } & \multicolumn{2}{|c|}{ Cleanrance } & Safety & Coordination \\
\hline \multirow[t]{2}{*}{ Effectiveness } & \multirow{2}{*}{$\begin{array}{c}\text { Transfer } \\
\text { Time }\end{array}$} & \multirow{3}{*}{$\begin{array}{l}\text { Queue } \\
\text { Length }\end{array}$} & \multirow[t]{2}{*}{ Density } & \multirow[t]{2}{*}{$\begin{array}{l}\text { Crowd Time } \\
\text { Distribution }\end{array}$} & $\begin{array}{c}\text { Time Safety } \\
\text { Level }\end{array}$ & Time-Flow \\
\hline & & & & & Evacuation & Facility-Flow \\
\hline Blanceness & Delay & & \multicolumn{2}{|c|}{$\begin{array}{l}\text { Crowd Space } \\
\text { Distribution }\end{array}$} & \begin{tabular}{|l} 
Time \\
$\begin{array}{c}\text { Space Safety } \\
\text { Level }\end{array}$
\end{tabular} & $\begin{array}{c}\text { Time- } \\
\text { Connection }\end{array}$ \\
\hline
\end{tabular}

Figure 4: Assessment indicator system of SRAIL.

indicators. Quantity indicators include density, flow, speed and queue length. Time indicators include time consumed at each trip step and the aggregate time of level of service at a specific region. Integrated indicators include level of service, comfort and station bottleneck position. These indicators can reflect facility usage, delay, congestion, safety and coordination. In order to assess the station more fully, a complete indicator system has been designed, which is shown in fig. 4.

Not all of these indicators are required in a simulation. This depends on what problem is faced and what problem causes most concern.

\section{Case study}

\subsection{Object station}

Beijing is a city with nearly 300 million inhabitants. There are six passenger train stations. As the first passenger dedicated railway station, Beijing south railway station connects Beijing and Tianjin city, which are the two most important cities in the north of China. The station opened before the 2008 Olympic Games. It has five floors with two metro line (M4\&M14) floors, one transfer floor, one platform floor with 24 tracks and one high level waiting floor with more than 20 waiting areas. After it became operational, the time taken to travel between Beijing and Tianjin decreased from 2 hours to $29 \mathrm{~min}$ and now takes 30 minutes. Every day, more than 162 trains depart from the station. It is one of the busiest railway stations in China. Nearly all of the high speed trains from Beijing depart from this station. After the M4 came into operation in October 2009, the passenger volume of the station was more than 55,000 per direction per day. An overview of the station is shown in fig. 5 .

\subsection{Simulation experiment}

Before the M4 was opened, the operators of the railway station needed an assessment of the capacity of passenger facilities. The utilization of the underground transfer hall should be evaluated after the line is opened. The highest passenger load of the station should be determined to decide the use of emergency plan.

To solve the problem, simulation experiments are designed. The key point is the underground transfer hall, so this floor should be paid much attention. 


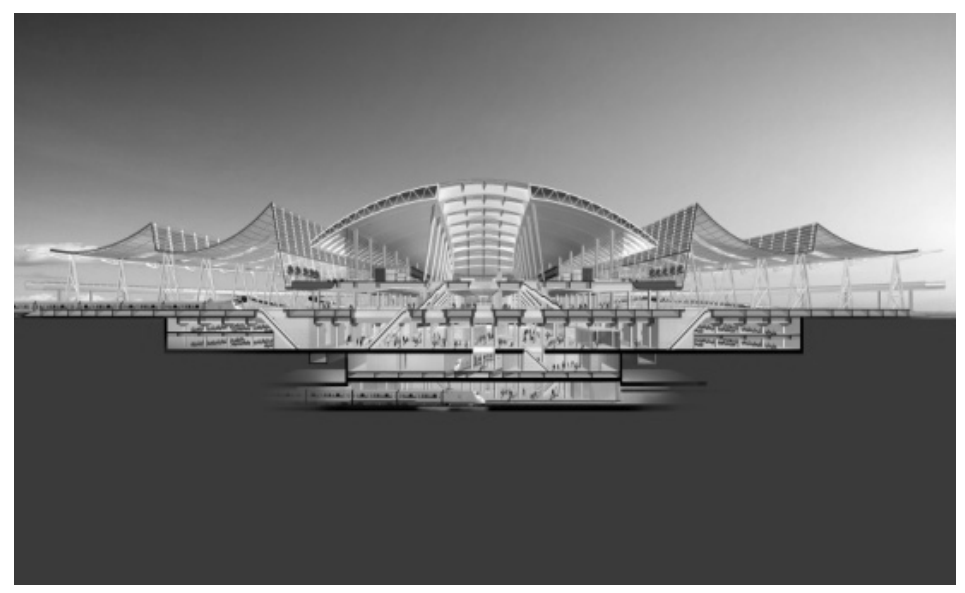

Figure 5: Overview of Beijing south railway station.

Table 2: $\quad$ Simulation schemes' parameters.

\begin{tabular}{|c|c|c|c|c|c|c|c|c|c|}
\hline \multirow[t]{2}{*}{ Scheme } & \multirow{2}{*}{$\begin{array}{l}\text { Train } \\
\text { number }\end{array}$} & \multicolumn{2}{|c|}{$\begin{array}{l}\text { Peak hour train } \\
\text { number }\end{array}$} & \multirow{2}{*}{$\begin{array}{l}\text { M4 } \\
\text { entran } \\
\text { ce }\end{array}$} & \multirow{2}{*}{$\begin{array}{l}\text { M4 } \\
\text { exit }\end{array}$} & \multicolumn{3}{|c|}{ Passenger arrive ratio } & \multirow{2}{*}{$\begin{array}{l}\text { Flow } \\
\text { cross } \\
\text { point }\end{array}$} \\
\hline & & $\begin{array}{l}\text { mornin } \\
\mathrm{g}\end{array}$ & Evening & & & M4 & Bus & Taxi & \\
\hline 1 & 70 & 10 & 8 & SNE & EW & 0.55 & 0.34 & 0.16 & 6 \\
\hline 2 & 100 & 20 & 12 & SNE & EW & 0.66 & 0.24 & 0.10 & 5 \\
\hline 3 & 150 & 30 & 22 & SNE & $\mathrm{W}$ & 0.50 & 0.34 & 0.16 & 5 \\
\hline
\end{tabular}

S: South; N: North; E: East; W: West

According to the current usage of the hall, the hall is divided into four zones: departure hall 1, departure hall 2, metro-rail transfer areas and others. In addition, in order to evaluate the highest passenger load, low, middle and high passenger volume schemes was designed. Three simulation schemes were designed according to different train numbers, passenger flow scale and operation method, as shown in table 2.

Other parameters are investigated and input into the model. The peak hours are selected (7:00-9:00 in the morning and 17:00-19:00 in the evening). It is assumed that all train occupancy is $100 \%$. According to the survey, passengers arrive at the station from 0 to 100 minute before train departure for long distance travel, because there are only a few trains per day. For short distance travel, passengers arrive at random. About $30 \%$ of passengers buy tickets before they arrive at the station. Station staff and people only at the station to greet people or buy tickets are not considered in this simulation. The delay of the train is randomly distributed, while all the trains should depart or arrive between 6:00 and 23:00. According to actual data, only platforms 2, 3 and 4 with six tracks could be used. On the second floor, two box offices (with a total of 28 service windows) are available. The desired speed of the passenger obeys the Gauss distribution $G(1.5,0.25)$. The passenger arrival probability of a train obeys the 
exponent distribution and the time spent when buying a ticket obeys the uniform distribution with $N(30,10)$ second. The simulation clock is set to 0.5 second. The ticket gate is only available 15 minutes before the train departs, and only correct ticket holders could pass the ticket gate. The ticket gate pass time is about 2 s per passenger. The simulation starts at 4:30 and ends at 23:59. The simulation is shown in fig. 6 .

\subsection{Result}

After simulation, the Instantaneous Maximum Passenger Number (IMPN) of the entire station and of each the concerned zones, Maximum Density (MD) and occupation time of Level of Service-A (LOSAT), is as recorded in table 3 and figs. 7 and 8 .

From the simulation result, it is found that the three schemes have the same peak time segment with train views. The time when the maximum passenger number appears, as well as the passenger volume in the station, is different. In scheme 1, the morning peak time is at about 8 a.m. with two peaks; the maximum passenger number is 3011 at 5:11 p.m. In scheme 2 , the morning peak comes earlier at 7 a.m. with three peaks. The maximum passenger number

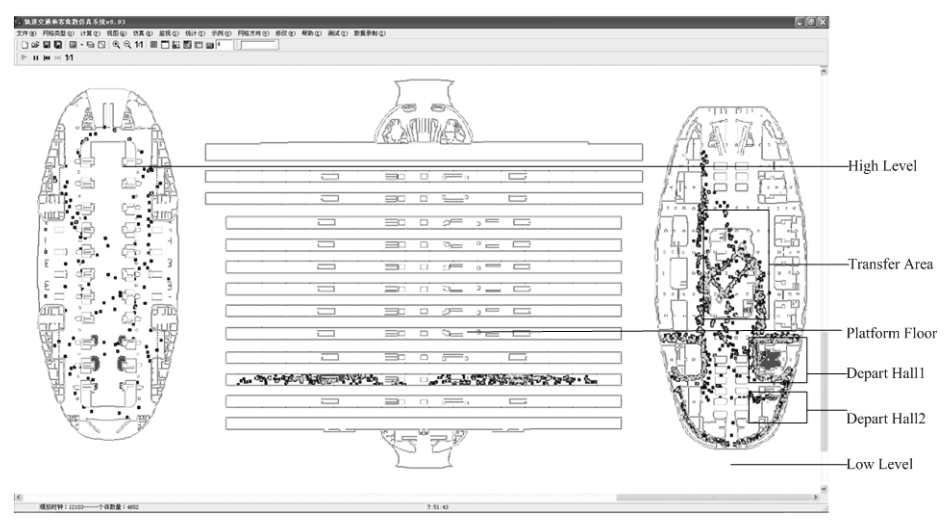

Figure 6: The instance density of the transfer hall.

Table 3: $\quad$ The statistics indicators.

\begin{tabular}{|c|c|c|c|c|c|}
\hline & Scheme 1 & Scheme 2 & Scheme 3 \\
\hline \multirow[t]{2}{*}{ Station } & \multicolumn{2}{|l|}{ IMPN } & 3011 & 3964 & 5134 \\
\hline & \multicolumn{2}{|c|}{ Crowd Point } & 2 & 4 & 3 \\
\hline \multirow{7}{*}{$\begin{array}{l}\text { Departure } \\
\text { hall }\end{array}$} & \multirow[t]{2}{*}{ IMPN } & Departure hall 2 & 788 & 1248 & 1324 \\
\hline & & Departure hall 1 & 660 & 1042 & 1064 \\
\hline & \multirow{3}{*}{$\begin{array}{l}\mathrm{MD} \\
(\mathrm{p} / \mathrm{m} 2)\end{array}$} & Departure hall 2 & 1 & 1.6 & 1.7 \\
\hline & & Departure hall 1 & 1.4 & 2 & 2.4 \\
\hline & & Transfer Areas & 0.025 & 0.058 & 0.06 \\
\hline & \multirow[t]{2}{*}{ LOSAT } & Departure hall 2 & $78.99 \%$ & $52.26 \%$ & $62.08 \%$ \\
\hline & & Departure hall 1 & $84.88 \%$ & $73.44 \%$ & $79.42 \%$ \\
\hline
\end{tabular}




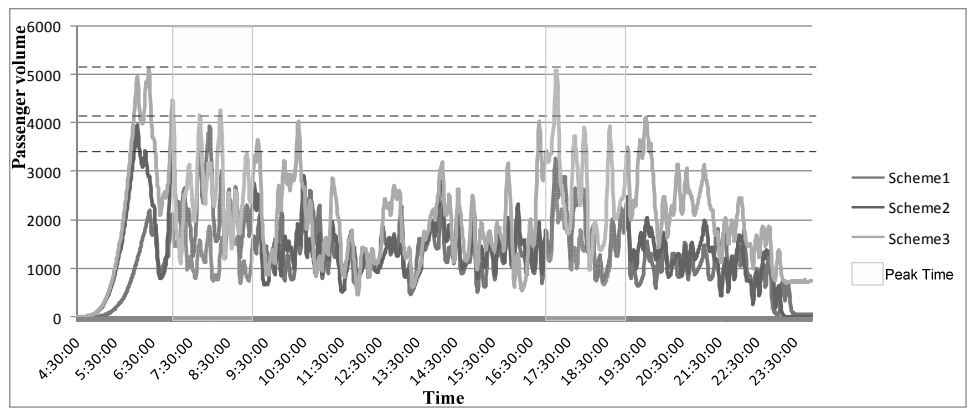

Figure 7: Instantaneous maximum passenger number.

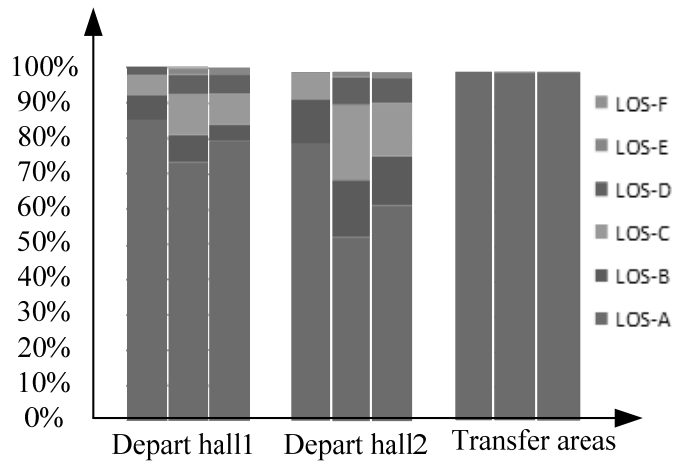

Figure 8: Level of service time occupation distribution.

appears at 6:06 p.m. with 3964 passengers. The morning peak time lasts longer than the evening peak time. In scheme 3 , the morning peak is about 6-7 a.m. and the maximum passenger number is 5134 at 6:14 p.m. The evening peak is stable in the three schemes at about 5:00 p.m. This result can be explained, as many long distance trains "depart at night and arrive early"; these cause the high density in the morning.

At departure halls, the instantaneous passenger number is also recorded. It is found that passenger volume rises very fast but reduces stage by stage. This might be because passengers who would go in many directions will share the same departing hall. Most of the passengers would like to gather in the underground departure hall 1. Passenger volume in departure hall 1 accounts for $65.8 \%$ of the total passengers in the underground. An interesting phenomenon is that the peak time of departure hall 1 is just the low volume time of departure hall 2. This is because of the uneven use of the departure halls. Departure hall 2 serves more tracks than departure hall 1. At the last scheme, the maximum density of departure hall 1 and departure hall 2 is $2 \mathrm{p} / \mathrm{m}^{2}$ and $1.6 \mathrm{p} / \mathrm{m}^{2}$; this is about 30 times the average density of the entire floor. One reason for this is that passengers take a rest and have to spend almost the longest time in the departure 
hall, another is the facility layout of this floor. This density is very close to the capacity of the facility, which means the station would be almost in saturation state under this level.

It is found that the crowd point in the three schemes is 2,4 and 3, respectively. Although the passenger volume is the highest in scheme 3 , the crowd point is not the most serious. This is because a special passenger flow line operation method is used in the last scheme. The mixed directional flow is changed into unidirectional flow by changing the escalator run direction.

Comparing the level of service occupation time of the facility, it is found that the level of service is not reduced very sharply with the increasing of the passenger volume. Even though the maximum density appears in departure hall 1, the level of service at departure hall 2 is worse than departure hall1 from the view of the whole day operation. This means the use of departure hall 2 is more balanced at this time.

\section{Conclusion}

A passenger simulation model and its implementation in China are described in this paper. A four steps model calibration and validation is presented for similar simulation applications. An indicator system is proposed to assess the station. A simulation tool, SRAIL, is developed based on the proposed model. SRAIL provides a user friendly interface and contains a lot of useful modules. The tool has been already used on station design tests, station egress capacity evaluations, passenger flow line improvements and station operation optimizations in many projects in China. An integrated simulation of station passenger flow and station yard operation is being studied and will be used in the future.

\section{Acknowledgements}

This work has been financed by the National Natural Science Foundation of China (NFSC), project ID: 60674012; National Key Technology R\&D Program (2009BAG12A10); Beijing Jiaotong University Research Fund, Project ID: 2007RC039. We would like to thank the related committee.

\section{References}

[1] Henderson, L.F., The statistics of crowd fluids. Nature, 229, pp. 381-383, 1971.

[2] Helbing, D., Keltsch, J. \& Molnár, P., Modelling the evolution of human trail systems. Nature, 388, pp. 47-50, 1997.

[3] Gipps, P. G., Marksjo, B., A Micro-simulation Model for Pedestrian Flows. Mathematics and Computers in Simulation, 27(2), pp. 95-105, 1985.

[4] Shigeyuki Okazaki, A Study of Simulation Model for Pedestrian Movement with Evacuation and Queuing. Proc. of the Int. Conf. on Engineering for Crowd Safety, eds. Roderick A. S. \&Jim F. D., Elsevier: London, pp. 271280, 1993. 
[5] Lovas G. G., Modeling and Simulation of Pedestrian Traffic Flow. Transportation Research B, 28B (6), pp. 429-443, 1994.

[6] Rothman D. H. \& Zaleski S., Lattice-gas models of phase separation: Interfaces, phase transitions, and multiphase flow. Reviews of Modern Physics, 66, pp.1417 1417-1479, 1994

[7] Helbing, D. \& Molnar, P., Social force model for pedestrian dynamics, Physical Review E, 51(5), pp.4282-4286, 1995.

[8] Hoogendoorn, S. P., Gas-Kinetic Modeling and Simulation of Pedestrian Flows. Transportation Research Record, 1710, pp. 28-36, 2000.

[9] Blue, V. J., Cellular Automata Model of Emergent Collective BiDirectional Pedestrian Dynamics. Proc. of the $7^{\text {th }}$ Int. Conf. on Artificial Life, eds. M.A. Bedau, J.S. McCaskill, N.H. Packard \&S. Rasmussen, MIT Press: Cambridge, pp. 437-445, 2000.

[10] Kirchner, A., Namazi, A., Nishinari, K. \& Schadschneider, A., Role of Conflicts in the Floor Field Cellular Automaton Model for Pedestrian Dynamics. Proc. of the $2^{\text {nd }}$ int. conf. on Pedestrians and Evacuation Dynamics. Eds. Edwin R. Galea E.R. U.K., pp. 51-62, 2003.

[11] Izquierdo, J. \& Montalvo, I., Forecasting pedestrian evacuation times by using swarm intelligence. Physica A. 7(388), pp.1213-1220, 2009.

[12] Maw, J. \& Dix, M., Appraisals of station congestion relief schemes on London Underground, Proceedings of PTRC Seminar, England, 335, pp.167-178, 1990.

[13] Gordge, R. M. \& Veldsman, A., Planning for pedestrians: Friendly, safe and viable transportation station environments, In: Freeman \& Jarnet, (eds.), Urban Transport Policy, Balkema, Rotterdam, Netherlands, 481487, 1998.

[14] Schelhorn, T., Sullivan, D. O, Haklay, M. \& Thurstain, M., STREETS: An agent based pedestrian model, Proc. of Computers in Urban Planning and Urban Management, Franco Angeli, Milano,1999

[15] Still G. K. Crowd Dynamics. PhD Dissertation, Warwick University, 2000.

[16] MacDonald M., STEPS Simulation of Transient Evacuation and Pedestrian Movements User Manual. Unpublished Work, 2003.

[17] Hoogendoorn, S.P. \& Bovy P.H.L., Pedestrian route-choice and activity scheduling theory and models, Trans. Res. Part B, 38, pp.169-190, 2004.

[18] Li, D., Modelling and Simulating Passenger Flow of Urban Railway Traffic, PhD Dissertation, Beijing Jiaotong University, 2007. 